\title{
Erros inatos do metabolismo no Hospi- tal das Clínicas de Ribeirão Preto. Um legado de múltiplas especialidades
}

\author{
Inborn Errors of Metabolism - a legacy of multiple specialties
}

Carolina A. R. Funayama

\begin{abstract}
RESUMO
Erros inatos do metabolismo (EIM) são doenças genéticas decorrentes, em sua maioria, de deficiências enzimáticas que levam a graves distúrbios metabólicos, e que vem sendo cada vez mais diagnosticados. No presente texto relata-se um histórico sobre a evolução da abordagem diagnóstica e tratamento de EIM no HCFMRP-USP.

Na FMRP-USP os EIM vêm sendo objeto de estudo em pós-graduação desde a década de 1980, dado o grande apelo clínico nas áreas de Genética Clínica, Pediatria e Neurologia Infantil, até então com auxílio do Laboratório de Patologia, além de convênio com a APAE de São Paulo para o diagnóstico de fenilcetonúria. Já iniciados os Programas de Pós-Graduação da FMRP-USP, a primeira tese a respeito foi realizada em 1980, sobre cistinúria, na Área de Genética; deficiência de glicose-6-fostato desidrogenase (G6PD) foi objetivo da segunda, em 1987, na de Pediatria. Desde essa época, garantem a rotina de investigação a realização da cromatografia de aminoácidos pelo Centro de Química de Proteínas da FMRP-USP, o convênio com o Serviço de Genética do HC de Porto Alegre-RS e a triagem urinária no Laboratório de Nutrologia, este, resultado de Mestrado em Neurologia em 1990. Nas duas últimas décadas vieram os ambulatórios especializados, o Programa de Triagem Neonatal, o tratamento por Reposição Enzimática e o apoio do Centro de Transplante de Células Tronco. Cabe ressaltar que até 2009, apenas mais uma tese foi apresentada. A perpectiva para o desenvolvimento dessa área é a consolidação de uma linha de pesquisa voltada exclusivamente para os EIM na FMRP-USP.
\end{abstract}

Palavras-chave: Erros Inatos do Metabolismo. Hospitais/história.

"Erros Inatos do Metabolismo" foi assim denominado pela primeira vez por Garrod ${ }^{1}$ em junho de 1908 no Croonian Lectures em Londres. Garrod ilustra seu conceito relatando quatro doenças: albinismo, alcapto- núria, cistinúria e pentosuria, fato este, que ficou reconhecido como um importante marco na compreensão das relações entre bioquímica, metabolismo e genética. Devido à baixa frequência populacional desse gru-
Livre-docente do Departamento de Neurociências e Ciências do Comportamento da Faculdade de Medicina da Universidade de São Paulo
Correspondência:

Prof ${ }^{-}$Dr ${ }^{a}$ Carolina A R Funayama Setor de Neurologia Infantil Departamento de Neurociências e Ciências do Comportamento Hospital das Clínicas da FMRP-USP

14048-900 Ribeirao Preto / SP carfunay@fmrp.usp.br

Artigo recebido em 08/04/2010 Aprovado em 20/09/2010 
po de doenças e da necessidade de progresso em recursos laboratoriais integrados nessas três áreas do conhecimento, o avanço nessa área foi muito lento nas décadas que se seguiram às observações de Garrod. No entanto, nas últimas décadas, os avanços nos recursos diagnósticos foram exponenciais, e os EIM, embora considerados raros, em seu conjunto, apresentam-se relativamente comuns na clínica prática, sendo estimados na América Latina em cerca de 1:1000 nascidos vivos. ${ }^{2}$ A grande diversidade de suas manifestações e a gravidade, expressando-se muitas vezes em emergência por hiperamonemia ou acidose, outras vezes latente, por deficiência mental, atraso no desenvolvimento ou regressão nas funções motoras ou mentais em qualquer idade, portanto, inclusive como demência, exigem preparo profissional com especialização nessa área, que engloba conhecimentos em bioquímica, genética, neurologia, nutrologia e gastrohepatologia pediátricas e em clínica médica. Envolve também a formação de profissionais em reabilitação e apoio para o seguimento dessas doenças, que de modo geral são crônicas e com especificidades no seu manejo.

Na FMRP-USP, nascia a primeira tese a respeito em 1980, sob a orientação da Prof ${ }^{a}$ Iris Ferrari no Departamento de Genética, primeira abordagem da cistinúria, tipos II e III na região de Ribeirão Preto, realizada pelo então doutorando, o médico Roberto Giugliani. $^{3}$

Logo, a fenilcetonúria despontou entre os erros com interesse no diagnóstico precoce com vistas a um tratamento específico para a prevenção da deficiência mental, uma vez que, sendo decorrente de um excesso de fenilalanina, um aminoácido essencial, poderia ser tratada com dieta industrializada, isenta desse aminoácido desde os primeiros dias após o nascimento.

Por essa época, a fenilcetonúria era investigada com o teste do cloreto férrico, uma gota sobre a urina na fralda de tecido, em recém-nascidos ou crianças maiores, quando a doença era lembrada pelo odor de gaiola de rato. Na década de 1980 houve expressivo avanço no diagnóstico de fenilcetonúria com o convênio com a APAE de São Paulo, trabalho pioneiro do Prof. Benjamin Schmidt na implementação do Teste do Pezinho. ${ }^{4}$ Nessa época, no HCFMRP-USP já se diagnosticavam as doenças de depósito, como as glicogenoses e doença de Gaucher com auxílio do Laboratório de Patologia da FMRP-USP, Professores José Alberto de Mello Oliveira e Sérgio Zucoloto e de Hematologia então sob a responsabilidade do Prof.
Roberto Passeto Falcão. As mucopolissacaridoses eram reunidas em setor ambulatorial do Serviço de Genética, então sob o comando do jovem Prof. João Monteiro de Pina Neto. O Departamento de Pediatria introduziu nesse período a triagem para G6PD, o segundo trabalho relacionado a um EIM, por Dr Paixão ${ }^{5}$, sob a orientação do Prof. Arthur Lopes Gonçalves e atualmente este exame encontra-se sob a responsabilidade do Laboratório de Hematologia.

Com a introdução das cromatografias de aminoácidos por troca iônica e de alta eficiência (HPLC) no Centro de Química de Proteínas sob a coordenação do Prof. Lewis Joel Greene, um convênio foi estabelecido em 1984 com o Serviço de Neurologia Infantil, criado pela Prof ${ }^{a}$ Maria Valeriana Leme de Moura Ribeiro, quando então as aminoacidopatias começaram a ser investigadas rotineiramente no HCFMRP-USP. Este trabalho foi ampliado posteriormente, em 1990 com a introdução da triagem urinária para EIM no Laboratório de Nutrologia sob a coordenação do Prof. José Ernesto dos Santos, após trabalho de triagem urinária de EIM dirigido para aminoácidos, mucopolissacaridoses e porfirias realizado pela Dr $^{a}$ Marlene de Fátima Turcato ${ }^{6}$, sob orientação da Prof ${ }^{a}$ Valeriana. Nessa época iniciou-se também intercâmbio entre os Serviços de Neurologia Infantil e o Serviços de Genética e Bioquímica do HC de Porto Alegre (1988 - Prof Moacir Wajner e Prof. Roberto Giugliani). A partir de então a detecção dos EIM vem sendo crescente, com a criação no final da década de 1990 de um ambulatório específico para atendimento de EIM, e em 2001 outro exclusivo para fenilcetonúria, ambos sob a responsabilidade da $\operatorname{Dr}^{\mathrm{a}}$ Marlene de Fátima Turcato, sendo este último com apoio do Laboratório de Triagem Neonatal implementado em 1994 pela Prof ${ }^{a}$ Lea Maria Zanini Maciel e, do Centro de Química de Proteínas, então sob a coordenação do Prof Greene, do Serviço Social e o de Nutrição e Dietética do HCFMRP-USP. Dr ${ }^{\mathrm{a}}$ Marlene Turcato tem coordenado eventos científicos na área de EIM, o terceiro em 2009, reunindo no HCFMRP-USP profissionais afins de expressão nacional e internacional, além de anualmente, juntamente com a equipe do Programa de Fenilcetonúria promover encontro dos pacientes com EIM nesta Instituição, com exposição de seus cardápios especiais.

Há cerca de 15 anos o HCFMRP-USP vem realizando reposição enzimática na Doença de Gaucher tipo I, processo que se inicia na Enfermaria de Gastropediatria sob a orientação da Prof ${ }^{a}$ Maria 
Inez Machado Fernandes e que, permanece desde 1998 no Serviço de Infusão do Hemocentro de Ribeirão Preto até hoje sob a coordenação da Dr ${ }^{a}$ Eugênia Ubiali, que acolheu, à época, iniciativa do Dr José Bernardo do Setor de Hematologia, que também assiste pacientes adultos. Mais recentemente, por intercorrência do Prof Pina, o Serviço de Infusão incluiu também as mucopolissacaridoses tipos I, II e VI. Novas perspectivas se abrem para o tratamento de EIM com a excelência do Serviço de transplante de Medula Óssea (TMO), sob a responsabilidade do Prof Julio César Voltarelli, hoje com importante avanço nos projetos com células tronco. Até o momento dois casos foram criteriosamente avaliados no Setor de TMO, sendo um com leucodistrofia metacromática e outro com adrenoleucodistrofia ligada ao X, havendo indicação do transplante de medula óssea. O primeiro ainda não poderia realizar o transplante nesta Unidade e foi encaminhado para o HC de Curitiba (PR), e o outro chegou a ser internado, mas seus distúrbios comportamentais impediram a continuidade do procedimento. Além desses, mais dois pacientes foram avaliados recentemente e aguardam doador.

Na presente década, agregam importante progresso na área de detecção e tratamento de EIM: em 2000, a implantação de recursos no Laboratório de Neurologia para investigação de doenças mitocondriais e projetos nesta área, pela Prof ${ }^{a}$ Cláudia Sobreira; em 2005, contratação como Docente FAEPA da Prof ${ }^{a}$ Regina Sawamura, que integra o trabalho de décadas com doenças hepáticas, desenvolvido pela Prof ${ }^{a} \mathrm{Ma}-$ ria Inez Machado Fernandes; contratação do Prof. José Simon Camelo Junior, que passou a ser responsável pela Divisão de Nutrição e Metabolismo, desenvolvendo linha de pesquisa em EIM, além da recente contratação da Prof ${ }^{a}$ Jaqueline Pontes Monteiro da área de Nutrição; em 2007, o Serviço de Genética do HC de Porto Alegre, sob a coordenação do Prof Roberto Giugliani passou a coordenar a Rede EIM Brasil, apoiada pelo SUS ${ }^{7}$, à qual o HCFMRP-USP passou a integrar na qualidade de "Centro Participante", com novas expectativas de consolidação de um serviço de detecção, tratamento e seguimento desse grupo de doenças.

Assim, considerando ser este ano o fechamento de um ciclo na história dos erros inatos do metabolismo, quando se comemorou em 2008 o centenário do trabalho pioneiro de Archibald Garrod e quando se prenunciam novos avanços com o apoio da Rede EIM Brasil, além do importante aporte de recursos huma- nos e laboratoriais no HCFMRP-USP, o objetivo deste trabalho é deixar aqui registrado o histórico desse atendimento.

Esse breve histórico da implementação do diagnóstico, atendimento e tratamento dos erros inatos no HCFMRP-USP evidencia as peculiaridades dessa área, que requer atenção, estratégias, serviço integrado, pode-se dizer interdisciplinar, senão transdisciplinar, à medida que se faz necessário um conhecimento comum.

As pesquisas sobre EIM nesse período de 1980 a 1990 incluem poucos estudos de relatos de casos, sendo um momento de tomada de conhecimento e caracterização de doenças que se manifestavam por retardo mental e se acenava com possibilidade de prevenção com dieta adequada, como ocorria à fenilcetonúria ou no caso das visceromegalias, as antigas tesaurismoses. Assim, foram a demanda ambulatorial, emergências e internações eletivas com desafios de difíceis diagnósticos, que os docentes e médicos assistentes das áreas de Neurologia Infantil, Genética Clínica, Pediatria da FMRP-USP e áreas laboratoriais vêm se dedicando ao estudo dessas doenças até a presente data. No quadro I sintetizamos os marcos dessa trajetória, cujos nomes representam suas equipes em cada época. O crescente número de casos confirmados frente ao total de suspeitos representa um esforço conjunto de profissionais das mais diversas regiões do Brasil, especialmente o sudeste, que fazem a suspeição e o encaminhamento, com aqueles que aqui recebem o paciente, na esperança de que o atendimento terciário possa cumprir o seu papel. Como hospital terciário caberá introduzir novas formas de prevenção, ou diagnóstico precoce dessas doenças, como a triagem neonatal ampliada ${ }^{8}$, utilizando-se o cromatógrafo MSMS, um recurso que, apesar de inicialmente de alto custo, torna-se barato frente ao gasto do Estado com educação e saúde da pessoa portadora de deficência no decorrer de sua vida, que hoje em dia chega à idade adulta.

Assim, também deve ser ressaltado para esse resultado o esforço individual de docentes e médicos assistentes junto a pesquisadores internacionais, a necessidade de exames realizados no exterior, que vem recebendo nos últimos anos importante apoio financeiro do HCFMRP-USP.

Deve-se também e principalmente à rotina de integração com o HC de Porto Alegre, que desde 1990 acolheu este serviço com interesses mútuos em pesquisa e na solução diagnóstica, sem contrapartida fi- 
nanceira, convênio este que em 2009 felizmente recebeu a participação do SUS, a partir da consolidação da Rede EIM Brasil. Esta, apoiada pelo Ministério da Saúde, é um projeto criado e coordenado pelo Prof Roberto Giugliani, um dos pioneiros na área de EIM no Brasil. A participação do HCFMRP-USP nessa Rede é imprescindível para o progresso dessa área, que requer formação de recursos humanos, com implementação de cursos de especialização e linhas de pesquisa com enfoque em projetos temáticos. Observa-se ao longo desses anos na FMRP-USP a falta de linhas de pesquisa voltadas exclusivamente para esse tema; apenas mais uma tese, da $\operatorname{Dr}^{\mathrm{a}}$ Marlene Turcato, em 1997, sob orientação do Prof Amilton Barreira foi apresentada. Assim, docentes com interesse específico em EIM são necessários, tanto em áreas clínicas junto ao paciente, como laboratoriais em bioquímica, neurologia, genética, hematologia e patologia, incluindo ainda novas perspectivas de tratamento, que envolvem setores como o de transplantes de células tron$\mathrm{co}^{10}$ e hepático ${ }^{11}$, assinalados com entusiasmo por centros afins já há algum tempo.

\section{Diagnóstico de Erros Inatos do Metabolismo HCFMRP-USP}

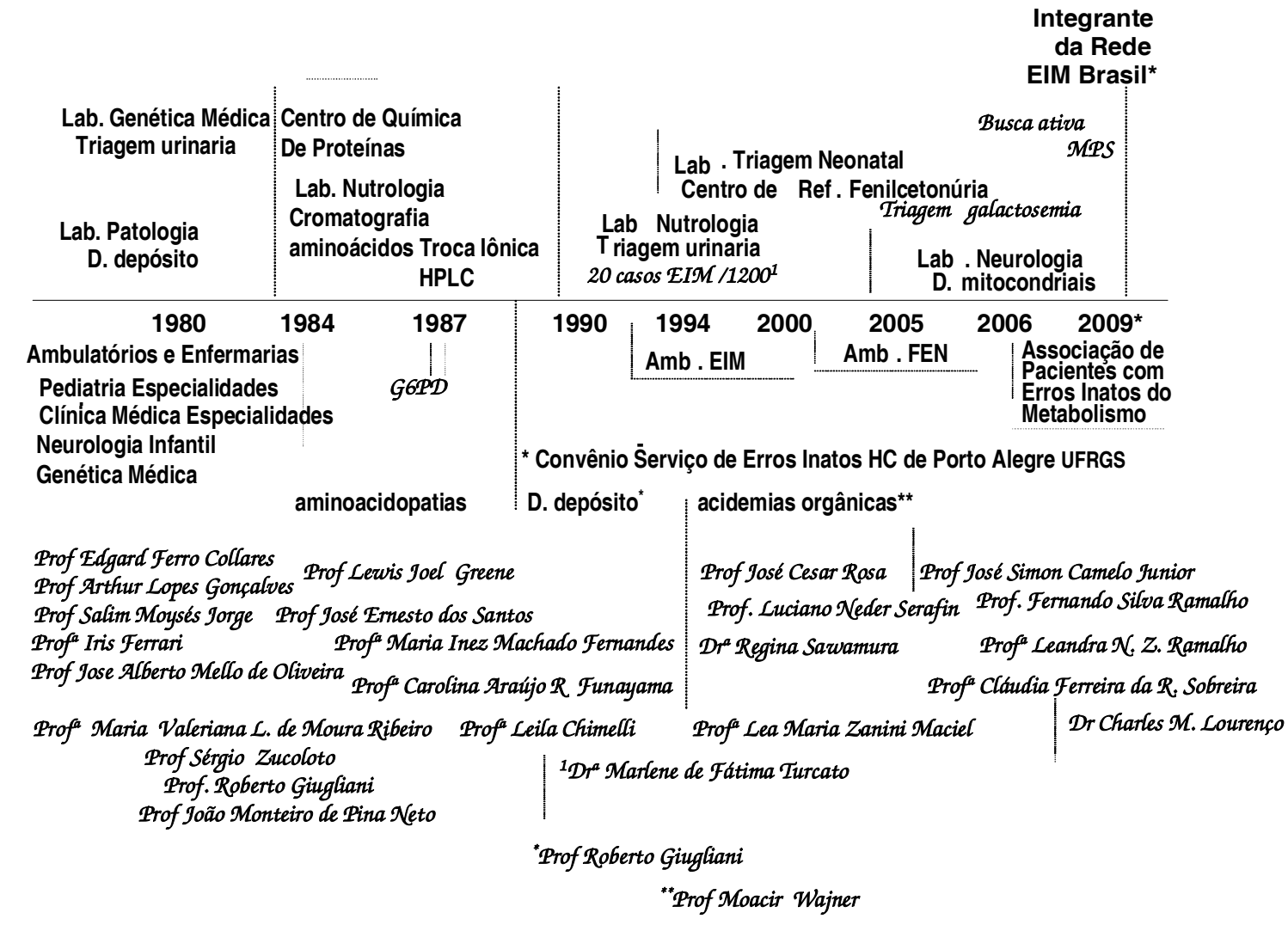

Quadro I - Recursos laboratoriais para diagnóstico de EIM no HCFMRP-USP e docentes e médicos assistentes das áreas envolvidas, de 1980 a 2009. 


\section{ABSTRACT}

Inborn errors of metabolism (IEM) are genetic diseases, mostly due to enzyme deficiencies leading to severe metabolic damages, increasingly diagnosed. The aim is to describe the history of the development of IEM diagnosis and treatment in the Hospital of Clinics of Ribeirão Preto, São Paulo University (HCFMRP-USP). At the beginnings of the Post-Graduate Programs in the School of Medicine of Ribeirão Preto of São Paulo University (FMRP-USP), the first thesis on IEM was performed in 1980 on cystinuria in the area of Genetics; G6PD goal was the second in 1987 in Pediatrics. Since that time, IEM diagnosis was possible, in the sequence, due to the chromatography of amino acids routine by the Center for Protein Chemistry, FMRP-USP in 1984, the partnership with the Department of Genetics, Hospital of Clinics of Porto Alegre. RS in 1988 and the urine screening in the Nutrology Laboratory of FMRP-USP, that resulted of a Master in Neurology in 1990. In the last two decades: the specialized outpatients clinics, the Program for Neonatal Screening, treatment by enzyme replacement and support of the Stem Cell Transplantation Center were implemented. It is noteworthy that by 2009, just one more thesis was presented. The perspective for the development of this area is the consolidation of a line of research focused exclusively on the EIM in FMRP-USP.

Keywords: Metabolism, Inborn Errors. Hospitals/history.

\section{Referências}

1. Scriver CR. Garrod's Croonian Lectures (1908) and the charter "Inborn Errors of Metabolism": Albinism, alkaptonuria, cystinuria, and pentosuria at age 100 in 2008. J Inherit Metab Dis 2008; 31: 580-98

2. Giugliani R, Coelho JC. Diagnóstico de erros inatos do metabolismo da América Latina. Braz J Gen 1997; 20: 147-54.

3. Giugliani, R. Heterozigose para cistinuria tipos II e III: estudo da metodologia de detecção, frequencia na regiao de ribeirao preto, sp, e relação com a formação de calculos urinarios. Tese. Faculdade de Medicina de Ribeirão Preto -USP:1980.

4. Freitas TNT. Fenilcetionuria e hipotireoidismo congenito diagnostico precoce e prevenção da deficiência mental. Niterói Universidade Federal Fluminense, EDUFF, 1990.

5. Paixão AC, Gonçalves AL, Tone LG, Jorge SM. Incidencia da Deficiencia da Enzima Glicose-6-Fosfato desidrogenase Em Recem Nascidos de Termo do Hospital das Clinicas da Faculdade de Medicina de Ribeirao Preto.. Jornal de Pediatria 1987; 62: 178-82

6. Turcato MF. Pesquisa de erros inatos do metabolismo através de testes de triagem urinários no Hospital das Clínicas da Faculdade de Medicina de Ribeirão Preto. 1990. Dissertação. Faculdade de Medicina de Ribeirão Preto, Ribeirão Preto, 1990.
7. Ministério da Saúde. Secretaria de Atenção à Saúde. Departamento de Atenção Especializada. Coordenação Geral de Média Complexidade Ambulatorial. Nota Técnica № 1551 de 01 de Dezembro de 2008: Política de Atenção Integral em Genética Clínica. In www.saude.gov.br acessado em 12 de dezembro de 2008.

8. Garg U, Dasouki M. Expanded newborn screening of inherited metabolic disorders by tandem mass spectrometry: clinical and laboratory aspects. Clin Biochem 2006;39: 315-32.

9. Turcato MF. Tirosinemia tipo II (Síndrome de Richner-Hanhart): estudo clínico, neurológico e bioquímico de cinco pacientes em uma família brasileira.. Tese. Faculdade de Medicina de Ribeirão Preto, 1997.

10. Boelens JJ, Prasad VK, Tolar J, Wynn RF, Peters C. Current international perspectives on hematopoietic stem cell transplantation for inherited metabolic disorders. Pediatr Clin North Am 2010;57: 123-45

11. Waelzlein JH, Puppi J, Dhawan A. Hepatocyte transplantation for correction of inborn errors of metabolism. Curr Opin Nephrol Hypertens 2009;18: 481-8 